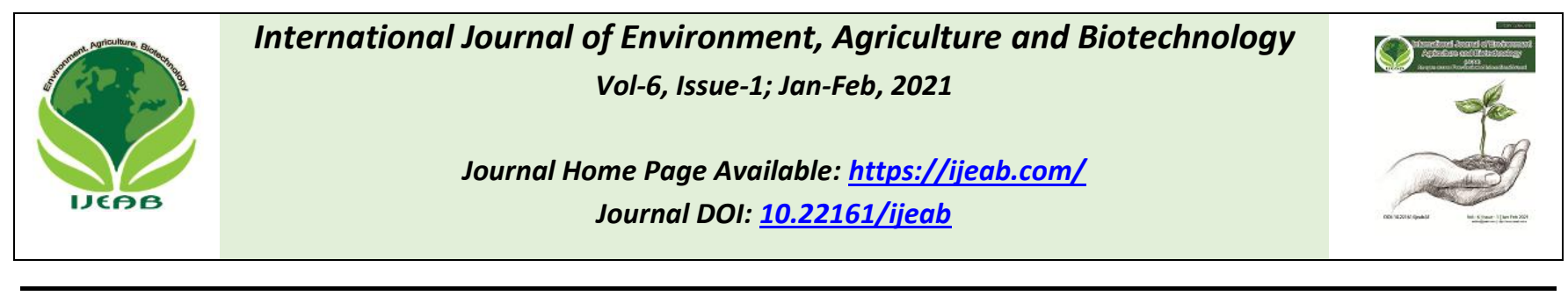

\title{
Metabolic Engineering of Microorganisms to Increase Production of Violacein
}

\author{
Nadia Sarwar ${ }^{1,2,3}$, Sarah Sarwar ${ }^{4}$, Sharoon Ejaz ${ }^{2}$, Abdulqader Al-Adeeb ${ }^{2}$, Waleed AL- \\ $\mathrm{Ansi}^{5,6}, \mathrm{Ye} \mathrm{Li}^{1,2,3, *}$, Zhonghu Bai ${ }^{1,2,3^{*}}$
}

\author{
${ }^{1}$ School of Biotechnology and National Engineering Laboratory for Cereal Fermentation Technology, Jiangnan University, Wuxi, China \\ ${ }^{2}$ School of Biotechnology and Key Laboratory of Industrial Biotechnology, Jiangnan University, Wuxi, China \\ ${ }^{3}$ Key Laboratory of Industrial Biotechnology, School of Biotechnology, Jiangnan University, Wuxi, China \\ ${ }^{4}$ Department of Biotechnology, Shaheed Benazir Bhutto University Peshawar, Pakistan \\ ${ }^{5}$ School of Food Science and Technology, State Key Laboratory of Food Science and Technology, Jiangnan University, 1800 Lihu \\ Avenue, Wuxi 214122, China. \\ ${ }^{6}$ Department of Food Science and Technology, Faculty of Agriculture, Sana'a University, Sana'a, Yemen. \\ *Corresponding Author
}

Received: 21 Nov 2020; Received in revised form: 28 Jan 2021; Accepted: 16 Feb 2021; Available online: 28 Feb 2021

(C)2021 The Author(s). Published by Infogain Publication. This is an open access article under the CC BY license (https://creativecommons.org/licenses/by/4.0/).

\begin{abstract}
Violacein, an indole derivative, is a violent pigment which is extracted from the bacteria. It is considered to be an important aromatic compound as it exhibits essential antiparasitic, antimicrobial and antitumoral characteristics. One of the most eminent derivatives that is being induced by the biosynthetic pathway of violacein is deoxyviolacein. However, it is produced in an insignificant amount. By expunging the VioD protein from the violacein pathway, deoxyviolacein can be generated which is devoid of a hydroxyl group. Another derivative that was produced in addition to deoxyviolacein is oxyviolacein, generated by the derivative of tryptophan i.e. 5-hydroxytryptophan. In this review, our main focus is on different engineered microorganisms in increasing the production of the violacein. On undergoing genetic analysis and determining the basic mechanism of violacein production showed that, violacein is formed by the condensation of 2 tryptophan molecules in presence of vioABCD gene cluster. However, later on, the presence of another gene vioE was revealed to be involved in violacein biosynthesis and a new pathway was suggested. McClean reported the involvement of quorum sensing mechanism via AHL's in violacein biosynthesis. Then using the above information and using violacein gene cluster vioABCDE, the violacein was produced in C. violaceum, Pseudoalteromonas sp. 520P1, V. natriegens, C. glutamicum, E. coli, Y. lipolytica and D. violaceinigra. Then the amount of violacein was increased by subjecting it to either batch or fed-batch fermentation. Then after its production, its anti-microbial activity was determined against Staphylococcus species. Also, its anti-cancerous activity was also determined on resistant leukemia cells.
\end{abstract}

Keywords - Violacein, Deoxyviolacein, Oxyviolacein, VioABCD.

\section{INTRODUCTION}

Metabolic engineering refers to the optimization of genetic and regulatory processes that occurs within the cell in order to increase the production of a specific substance in the cells. It includes series of biochemical reactions together with the enzymes to convert the raw materials into that specific substance and increase its amount. Metabolic engineering specifically deals with creating a mathematical model of these pathways, calculating its yield and blocking the path that will constrain the production of our desired substance (Yang, Bennett, \& San, 1998). In this review, our main focus is on different engineered microorganisms in increasing the production of the violacein. 
Violacein, an indole derivative, is a violent pigment which is extracted from the bacteria. It is considered to be an important aromatic compound as it exhibits essential antiparasitic, antimicrobial and antitumoral characteristics (Durán et al., 2007). Being a bisindole, it is produced by a vast genus of bacterial strains which includes: Microbulbifer sp., Pseudoalteromonas, Collimonas and Duganella etc. These strains are phylogenetically varied and thus, are isolated from different locales like the rhizosphere of olive groves (Aranda, Montes-Borrego, \& Landa, 2011), within glaciers (Avguštin, Bertok, \& Avguštin, 2013) and even from sea sponges surfaces. Among the genus of violacein-producing strains, Chromobacterium is the best one i.e. Chromobacterium violaceum (Durán \& Menck, 2001). Violacein as a secondary metabolite is associated with the production of biofilm. Quorum sensing mechanisms modulate its production within $\mathrm{C}$. violaceum and other bacterial strains (McClean et al., 1997). Due to its facile visualization, its production has become an effective criterion of quorum sensing substances and their inhibitors (Burt, OjoFakunle, Woertman, \& Veldhuizen, 2014).

\section{OXYVIOLACEIN AND DEOXYVIOLACEIN}

Despite grabbing most of the attention, violacein is not only the substance produced within the bacterial hosts by proteins i.e. VioA, VioB, VioC, VioD and VioE. One of the most eminent derivatives that is being induced by the biosynthetic pathway of violacein is deoxyviolacein. However, deoxyviolacein, in comparison to violacein, is produced in an insignificant amount in Janthinobacterium lividum (Rodrigues et al., 2012).
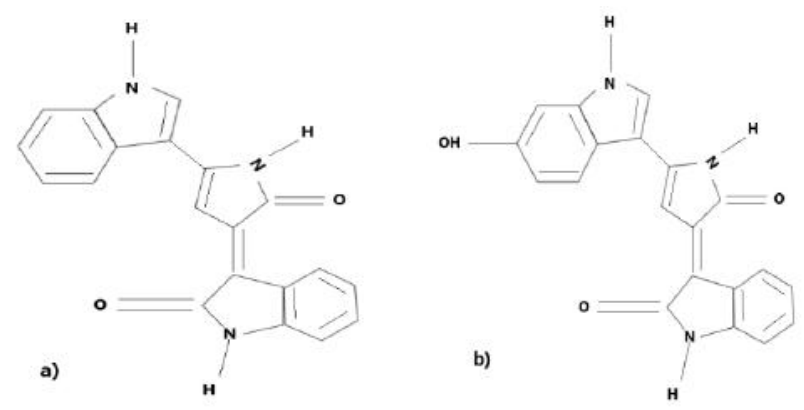

Fig.1: Chemical structures of a) deoxyviolacein and b) violacein

Compatible results were also depicted in research with Duganella sp. B2 where also deoxyviolacein was produced in low concentration as compared to violacein which was evaluated by HPLC (Jiang et al., 2012). Crude violacein extracts that are procured from the natural bacterial strains contain around $\sim 10-20 \%$ of deoxyviolacein with $85 \%$ mostly violacein on the basis of HPLC evaluation.
By expunging the VioD protein from the violacein pathway, deoxyviolacein can be generated which is devoid of a hydroxyl group. A recent study by Xing group divulged the elevated production and characterization of deoxyviolacein using the chimeric Citrobacter freundii having a plasmid with VioD gene knocked out of it. Their research disclosed that deoxyviolacein showed comparatively better photostability as compared to the violacein under tests with either UV or natural light. However, both were proved to be toxic when are exposed to the $24 \mathrm{~h}$ toxicity tests with HepG2 cell lines. However, deoxyviolacein impact was dose-independent as compared to violacein i.e. dose-dependent. The variance between violacein and deoxyviolacein was more conspicuous when the viability of HepG2 cell lines was determined after $48 \mathrm{~h}$ (Jiang et al., 2012).

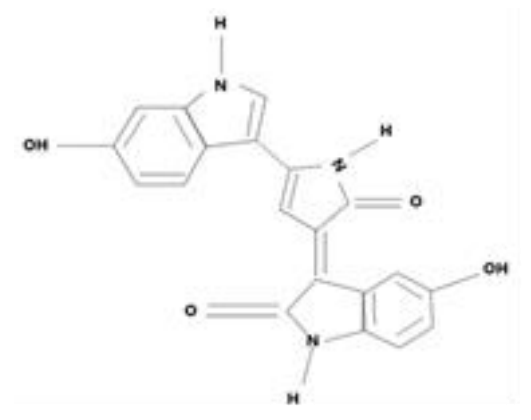

\section{Fig.2: Chemical structure of oxyviolacein.}

Another derivative that was produced in addition to deoxyviolacein is oxyviolacein, generated by the derivative of tryptophan i.e. 5-hydroxytryptophan (Sánchez, Braña, Méndez, \& Salas, 2006). In oxyviolacein, there is an extra hydroxyl group as compared to deoxyviolacein that lacks the hydroxyl group. This presence of extra hydroxyl group has elevated its efficiency against human pathogens like Staphylococcus aureus that was reduced in deoxyviolacein as it is devoid of its hydroxyl group (H. Wang et al., 2012).

\section{GENETIC ANALYSIS AND THE BASIC MECHANISM OF VIOLACEIN BIOSYNTHESIS}

An intermediate of glycolysis i.e. phosphoenolpyruvate (PEP) and an intermediate of Hexose monophosphate pathway (HMP) i.e. erythrose-4-phosphate (E4P) together initiates the biosynthesis of the aromatic amino acids that leads through various steps to produce chorismate that lead to a branch that commences with anthranilate that finally leads to tryptophan pathway. The defective mutants in the HMP pathway can produce an enhanced mount of E4P that act as a limiting substance in the biosynthesis of violacein (Ikeda \& Katsumata, 1999). 
Previous studies on the violacein biosynthetic pathway claimed 5-hydroxy-L-tryptophan to be the precursor in violacein synthesis (Hoshino \& Ogasawara, 1990). However, it was revealed later that two trp molecules generate all the $\mathrm{C}, \mathrm{N}$ and $\mathrm{H}$ atoms while molecular $\mathrm{O}_{2}$ generates the $\mathrm{O}$ atoms of the violacein (Antonio, Haun, \& Pilli, 1994) (Momen \& Hoshino, 2000). It is now perceptible that 2 trp molecules form a fundamental structure of violacein which is accompanied by the indole ring oxygenation of the intermediate. Like in E. coli, anthranilate biosynthesis initiates the biosynthesis of tryptophan which is encrypted by various genes i.e. $\operatorname{trp} A$, $\operatorname{trp} B, \operatorname{trp} C, \operatorname{trp} D, \operatorname{trp} E, \operatorname{trp} F$ and $\operatorname{trp} G$. However, unlike $E$. coli, they are not categorized into an operon. Instead, they concoct clusters with genes not involved in tryptophan biosynthesis. August and his co-workers cloned and sequenced the complete operon of violacein biosynthesis. It was disclosed that VioA, VioB, VioC and VioD genes constitute the violacein biosynthetic operon genes as shown in figure 3 and table 1.

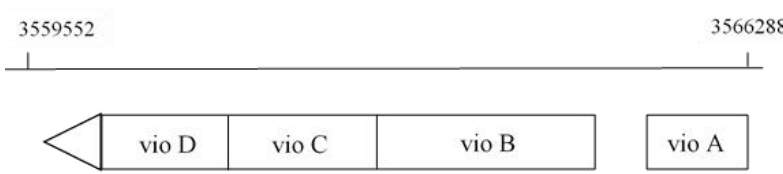

Fig.3: Structural gene operon of violacein biosynthesis

After cloning and sequencing the operon for biosynthesis of violacein, these scientist designed a model biosynthetic pathway showing the imputed activities of every gene product in this pathway (figure 4) (August et al., 2000).

Table 1: Major ORF's (open reading frames) for violacein metabolism and expression (de Vasconcelos et al., 2003)

\begin{tabular}{ccc}
\hline ORF name & ORF number & ORF product name \\
\hline vioA & CV3274 & Tryptophan-2-monooygenase \\
vioB & CV3273 & Polyketide synthase \\
vioC & CV3272 & Monooxygenase \\
vioD & CV3271 & Hydroxylase \\
$\operatorname{trpA}$ & CV2761 & Tryptophan synthase, alpha subunit \\
$\operatorname{trpB}$ & CV2762 & Tryptophan synthase, beta subunit \\
$\operatorname{trpC}$ & CV2712 & Indole-3-glycerol phosphate synthase \\
$\operatorname{trpD}$ & CV2173 & Anthranilate \\
$\operatorname{trpE}$ & CV2179 & Anthranilate synthase component-I \\
$\operatorname{trpF}$ & CV2763 & Phosphoribosylanthranilate \\
& & isomerase
\end{tabular}

The vioA, vioC and vioD gene products showed similarity with the nucleotide-dependent monooxygenases. Research on violacein biosynthesis suggested that while the hydroxylation of one tryptophan molecule is catalyzed by vioD gene, the oxidative deamination of the second trp molecule is catalyzed by vioA gene and similarly, intermediate oxidation of violacein is catalyzed by vioC. Similarly, vioB protein was found to be compatible with polyketide synthase, an enzyme that exhibits an amazing activity i.e. its capability to catalyze non-ribosomal peptidal bonds and in the biosynthesis of violacein, it mediates the condensation of 2 trp derivative molecules that are important for pigment production (Antônio \& Creczynski-Pasa, 2004).

\section{VIOLACEIN AND DEOXYVIOLACEIN BIOSYNTHESIS IN C. VIOLACEUM}

Till now, it was believed that in violacein biosynthesis, only four genes (vioABCD) were involved (August et al., 2000) (Brady, Chao, Handelsman, \& Clardy, 2001).

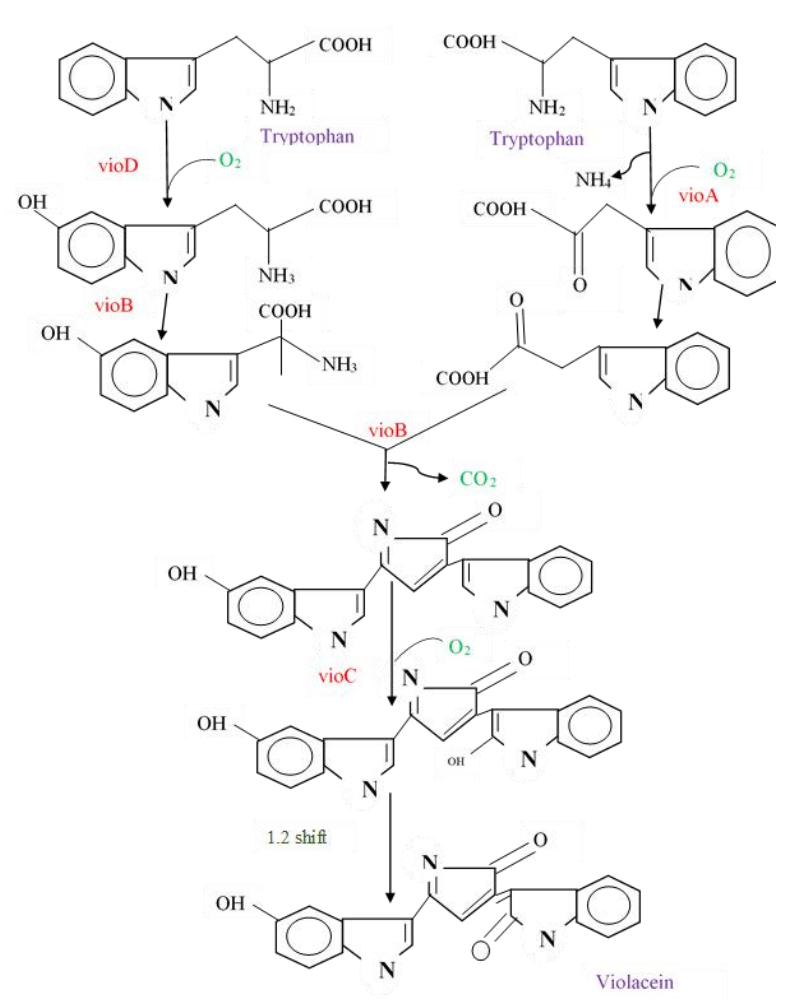

Fig.4: Violacein biosynthetic pathway suggested by August et al. 2000.

However, whole-genome sequencing of Chromobacterium violaceum in 2003 disclosed the existence of the $5^{\text {th }}$ gene in the gene operon of violacein (de Vasconcelos et al., 2003). Thus, until the role of this $5^{\text {th }}$ gene was not revealed, the complete violacein biosynthetic path couldn't be identified (Sánchez et al., 2006) (Balibar \& Walsh, 2006). It was revealed that the 
product of $5^{\text {th }}$ gene, vioE protein catalyzes the protodeoxyviolacenic acid formation from an unidentified intermediate, compound X (Asamizu, Kato, Igarashi, \& Onaka, 2007) (figure 5). Thus, it was evident that vioE proved to be requisite for the formation of violacein structure. Recent studies have reported the comprehensive structure and reaction process of the VioE protein. The five violacein biosynthetic enzymes i.e. VioABCDE were encoded by 5 genes arranged in a single operon i.e. vioABCDE (Hirano, Asamizu, Onaka, Shiro, \& Nagano, 2008) (Ryan, Balibar, Turo, Walsh, \& Drennan, 2008). These 5 enzymes VioABCDE were cloned and expressed under invitro conditions by Balibar and Carl who reported L-tryptophan as a precursor of violacein instead of 5hydroxy-L-tryptophan. The pathway model suggested for violacein and deoxyviolacein biosynthesis is shown in figure 5 .

Oxidation of tryptophan is catalyzed by VioA. The coupling of 2 IPA imine molecules to generate an unidentified intermediate $\mathrm{X}$ is the responsibility of VioB. Protodeoxyviolaceinic acid is produced by the newly discovered enzyme, VioE. Inevitably, oxygenation of indole rings at 2 and 5 positions to form violacein is catalyzed by VioC and VioD respectively. Oxygenation of indole ring at position 2 by $\mathrm{VioC}$ from protodeoxyviolaceinic acid generates deoxyviolacein, the by-product of violacein (Hirano et al., 2008).

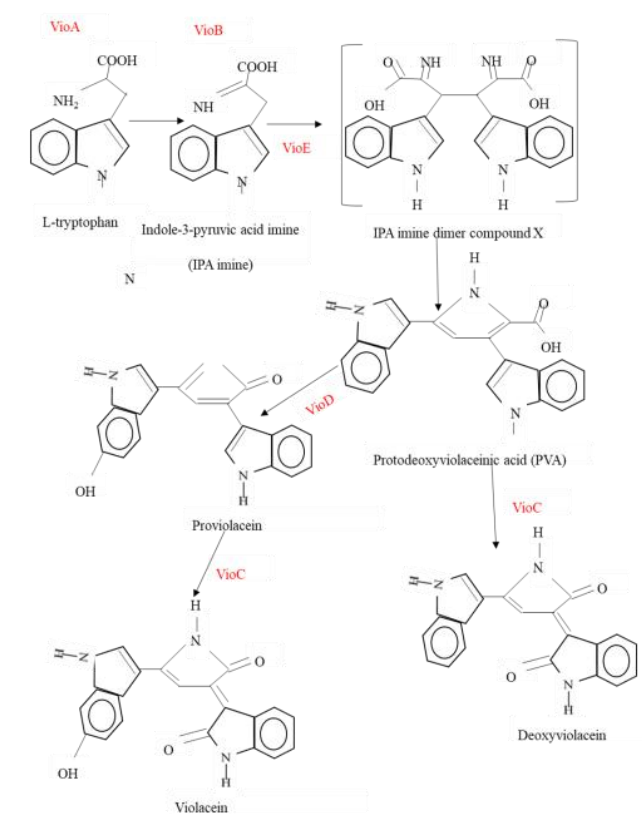

Fig.5: Violacein and deoxyviolacein biosynthesis pathway in $C$. violaceum

\subsection{QUORUM SENSING MODULATION OF SECONDARY METABOLITES}

Quorum sensing processes via autoinducer molecules that the bacteria secrete modulates the production of violacein in C. violaceum. Quorum sensing is a mechanism that bacteria used in order to communicate with each other by releasing signaling substances (Waters \& Bassler, 2005) (Williams, Winzer, Chan, \& Camara, 2007). This quorum sensing mechanism regulates the production of many secondary metabolites like pigments, toxins, antibiotics, bioluminescence and biofilm formation.

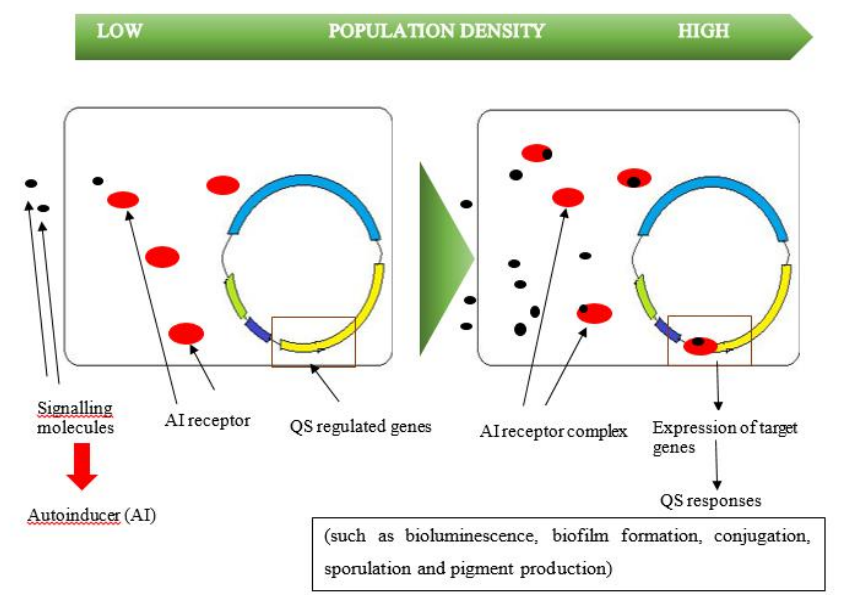

Fig.6: Quorum sensing mechanism

\subsection{QUORUM SENSING IN VIOLACEIN BIOSYNTHESIS}

It was reported by McClean that quorum sensing via AHLs i.e. N-acyl homoserine lactones regulates the production of violacein in $\mathrm{C}$. violaceum (figure: 7).

It was suggested that the cumulated AHL forms a complex by binding with receptor proteins which then activate the expression of gene cluster by interacting with transcriptional regulator site of violacein operon, which eventually leads to the synthesis of violacein. This alleged transcriptional promoter site is present in the intervening area between gene vioA and its upstream adjoining protein gene. There is little evidence for the location of quorum sensing regulatory sites (McClean et al., 1997). 


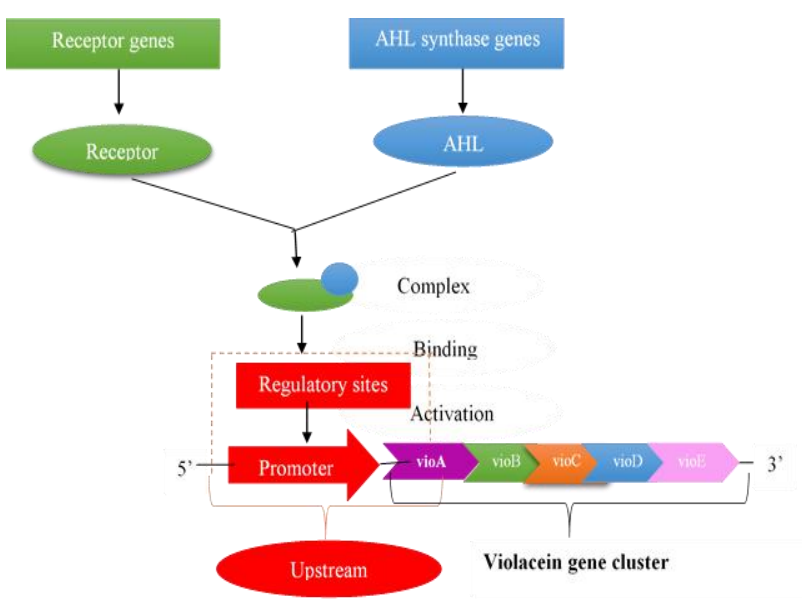

Fig.7: Quorum sensing model of regulation of violacein gene regulation in $C$. violaceum.

\section{VIOLACEIN PRODUCTION AND REGULATION IN PSEUDOALTEROMONAS SP. 520P1}

Zhang conducted an experiment to demonstrate the production and regulation of violacein by Pseudoalteromonas sp. 520P1 under quorum sensing systems via AHLs. This 520P1 strain is a gram-negative bacteria which was sequestered from the seawater of Cap Muroto in Japan (Yada et al., 2008). However, this strain showed violacein production under static culture circumstances only. However, previous studies showed that the 520P1 strain can produce violacein under the regulation of the quorum sensing process through AHLs (Y. Wang et al., 2008). In this research, they characterized and cloned the gene cluster of violacein and its upstream region using a fosmid library. It was used to create stable libraries using complex genomes (Huang et al., 2009) and sequestering genes (Schloss et al., 2010) from the genomic DNA. It contained 13,000 clones developed from the 520P1 strain's genomic DNA. 5 clones were isolated containing violacein gene clusters. Thus, 5 ORF's cluster i.e. vioABCDE of 7383 total lengths for the biosynthesis of violacein was obtained. A highly conserved sequence was determined in 520P1 strain at 200bp upstream of gene cluster having promoter sequences i.e. -10 and -35 box. $\sim 700 \mathrm{bp}$ downstream and $\sim 1500 \mathrm{bp}$ upstream are sequences that encodes for 2 putative proteins (figure: 8 ).

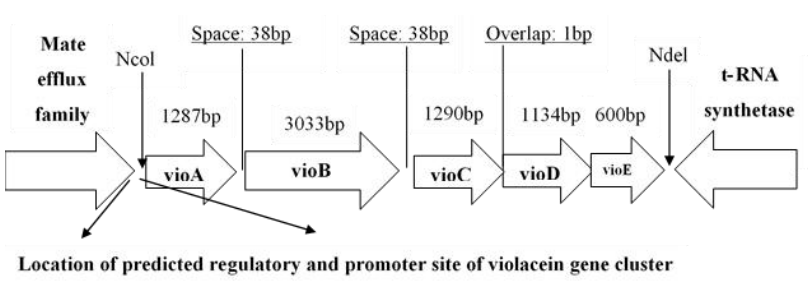

Fig.8: Schematic diagram of Pseudoalteromonas sp. $520 P 1$ strain gene cluster

Heterologous expression of gene cluster was accomplished using a recombinant pET vector in $E$. coli to analyze its ability to produce violacein (figure: 9). Successful violacein gene cluster expression of 520P1 strain in $E$. coli was confirmed by the emergence of violet colonies of recombinant $E$. coli.

However, the violet color indicating violacein production appears only when recombinant $E$. coli were incubated in absence of IPTG inducer at $50 \mathrm{rpm}$ at $20^{\circ} \mathrm{C}$. HPLC and UV-VIS spectrum analysis confirmed violent pigment produced by recombinant $E$. coli to be identical to violacein. Normally, 520P1 strain produced the mixture of violacein and deoxy violacein, but, elution profile in HPLC analysis indicated a single peak showing the production of the only violacein in recombinant $E$. coli (ZHANG, 2010).

\section{HETEROLOGOUS BIOSYNTHESIS OF VIOLACEIN IN VIBRIO NATRIEGENS}

\subsection{VIOLACEUM BIOSYNTHETIC PATHWAY CONSTRUCTION IN V. NATRIEGENS}

Ellis et al in 2019 conducted a research to determine the feedstock flexibility and heterologous natural product (violacein) production in $V$. natriegens using the gene cluster of C. violaceium. V. natriegens only encodes for the precursor of tryptophan, thus, can't encodes the violacein biosynthesis (Lee et al., 2019). 


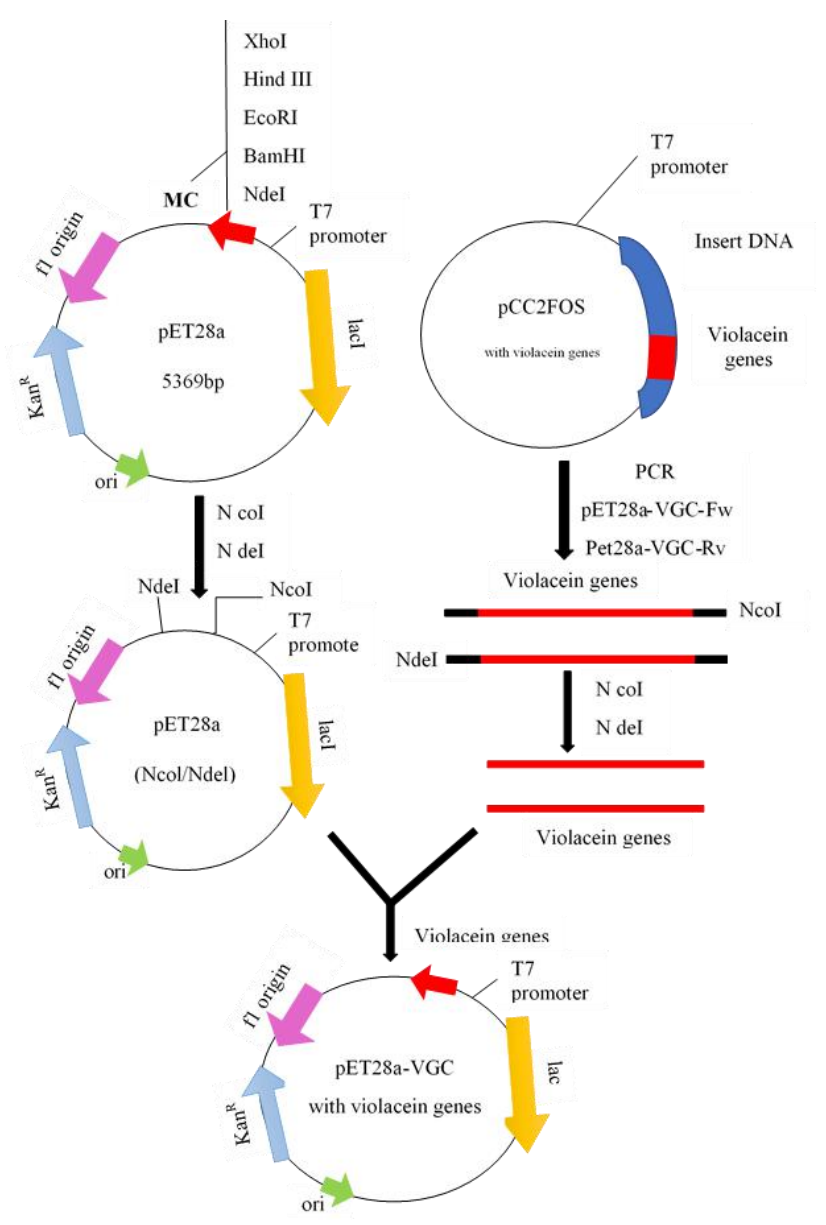

Fig.9: Schematic diagram of recombinant vector construction containing violacein gene cluster

Tryptophan is produced from basically two pathways in $V$. natriegens i.e. in glycolysis form phosphoenolpyruvate (PEP) and in pentose phosphate pathway from erythrose4-phosphate (E4P) (figure:10).

Table 2: Reported heterologous genes for constructing violacein synthetic pathway in V. natriegens (Kim et al., 2016) (M. E. Lee et al., 2013)

\begin{tabular}{|c|c|c|}
\hline Gene & Gene ID & Protein \\
\hline vioA & 24947400 & L-tryptophan synthase \\
\hline vioB & 24945600 & $\begin{array}{l}\text { Iminophenyl-pyruvate dimer } \\
\text { synthase }\end{array}$ \\
\hline vioC & 24948167 & Violacein synthase \\
\hline vioD & 24947515 & Tryptophan hydroxylase \\
\hline vioE & 24949508 & $\begin{array}{l}\text { Protodeoxyviolaceinate } \\
\text { monooxygenase }\end{array}$ \\
\hline
\end{tabular}

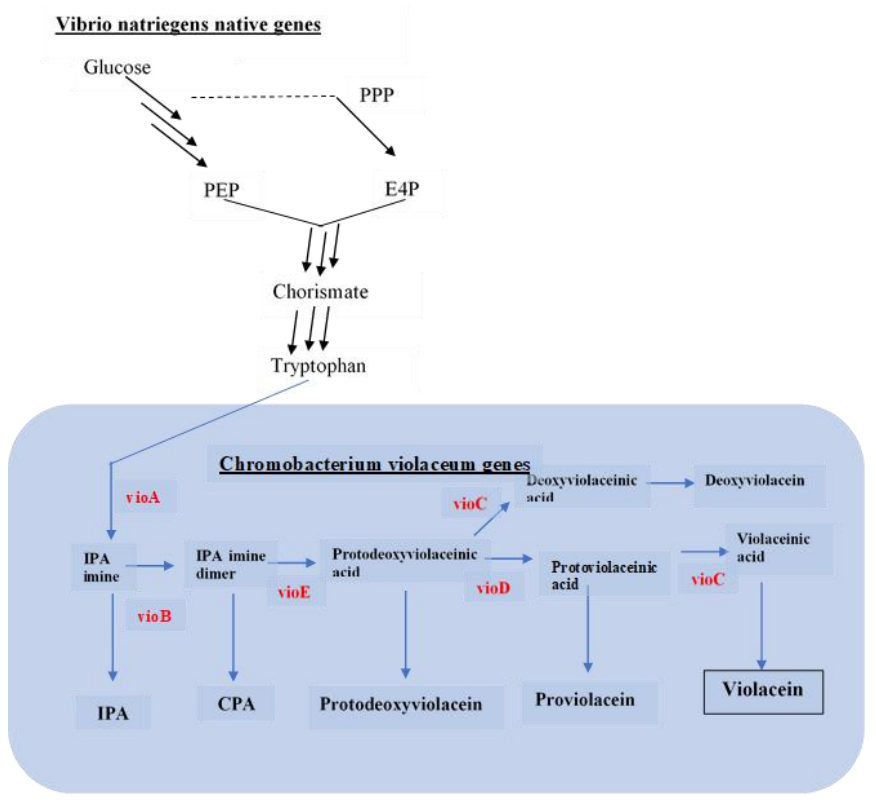

Fig.10: Violacein biosynthetic natural and heterologous pathways in V. nitrogens. Plasmid ( $p$ Vio) map or violacein biosynthesis

As described above, there is the involvement of 5 enzymes for the conversion of tryptophan to violacein. It was also disclosed that the violacein biosynthetic pathway consists of various opportunities to convert intermediates non-enzymatically to terminal co-products, limiting the overall yield as seen in figure 10. Genes, gene ID and enzyme names (Jiang, Wang, Zhang, Lou, \& Xing, 2010) (Dantas, Volpe, Durán, \& Ferreira, 2012) are listed in the table 2. For the production of violacein, a plasmid (pVio) was constructed which contained violacein pathway gene cluster (vioABCDE) from C. violaceum ATCC 12472 (figure: 11).

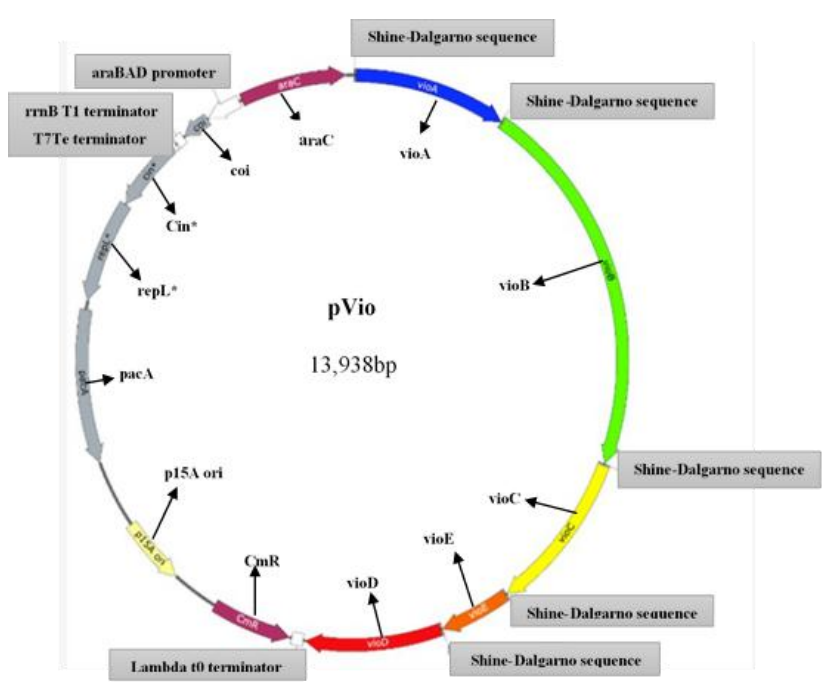

Fig.11: Plasmid (pVio) map or violacein biosynthesis 


\subsection{FEEDSTOCK FLEXIBILITY AND HETEROLOGOUS PRODUCTION OF VIOLACEIN BIOSYNTHESIS}

$V$. natriegens having plasmid (pVio) was then tested in both minimal media and LBv2 rich media consisting of various carbon sources required for the biosynthesis of violacein and deoxyviolacein (by-product). UV-HPLC analysis showed that in rich media, $V$. natriegens produced $13.1 \pm 0.9 \mathrm{mg} / \mathrm{l}$ and $24.9 \pm 3.1 \mathrm{mg} / \mathrm{l}$ of violacein and deoxyviolacein respectively. It was observed that a higher amount of violacein is produced in mannitol followed by glucose, fructose and $\mathrm{N}$-acetyl-glucosamine when absorbance is determined at $\mathrm{OD}_{600}$. A similar amount of violacein is produced in both LBv2 rich media and minimal media with $15.5 \mathrm{mg} / \mathrm{l}$ mannitol. However, the deoxyviolacein to violacein ratio was very interesting. Rich media produced $1.9 \mathrm{X}$ more deoxyviolacein than violacein but, more violacein is produced as compared to deoxyviolacein i.e. by $4.2 \mathrm{X}$ in minimal media with different carbon sources. Another significant finding was the exclusion of minimal media + arabinose for violacein biosynthesis because the findings will most likely to be confuted due to induced arabinose synthesis by phagemid-elements present in pVio plasmid. Violacein production by transformant containing $\mathrm{pVio}$ plasmid is comparatively less as compared to other carbon sources due to the synthesis of these proteins or lysis of $V$. natriengens due to activation of prophages (Ellis et al., 2019).

\section{VIOLACEIN HYPER-PRODUCTION FROM ENGINEERED CORYNEBACTERIUM GLUTAMICUM}

Because of the numerous advantages of Corynebacterium glutamicum as a microbial cell factory, it is basically identified as safes as compared to E. coli. C. glutamicum has dominated the fermentation processes of industrialscale to synthesize various amino acids and other products for food, animal feed, cosmetics and health (Pühler, Kalinowski, \& Tauch, 2008). One of the welldeveloped hyper-producer of L-tryptophan is ATCC 21850 strain of C. glutamicum. Since L-trp is the direct precursor of crude violacein biosynthesis, this strain has provided an attractive platform. For violacein biosynthesis, L-tryptophan producing and wild type strains i.e. ATCC 13032 of C. glutamicum has been engineered as novel carcass (Heery, Fitzpatrick, \& Dunican, 1994). Expression vectors of $E$. coli/ $C$. glutamicum shuttle (pEC-XK99E) (Jakoby, Krämer, \& Burkovski, 1999) have provided a plasmid backbone for the construction of functional plasmids. For the construction of plasmid, E. coli DH5 $\alpha$ was used using golden-gate DNA assembly process (Hillson, ISSN: 2456-1878
Rosengarten, \& Keasling, 2012) and this plasmid was then transformed into C. glutamicum strains i.e. ATCC 21850 or ATCC 13032 (Tauch et al., 2002). As a metabolic engineering carcass, ATCC 21850 strain is used as a novel host as it produced $162.98 \mathrm{mg} / \mathrm{l}$ of Ltryptophan. In ATCC 21850 strain having key promoter, heterologous vio operon from $C$. violaceum was hyperexpressed to produce $532 \mathrm{mg} / \mathrm{l}$ of violacein. Keeping the violacein toxicity in consideration, inducible promoters were used while expression of vio operon and $629 \mathrm{mg} / \mathrm{l}$ of violacein was synthesized under batch culture conditions. The compressed RBS of vio genes were exchanged with whole strong C. glutamicum because of vio operon's economical coding nature. And synthetic operon was developed by assembling extended expression units. Using this techniques, $1116 \mathrm{mg} / \mathrm{l}$ of violacein was synthesized under batch culture conditions. Fermentation temperature, culture composition, induction time and induction concentration was studied to optimize the fermentation process and consequently, in $3 \mathrm{~L}$ bioreactor, $47 \mathrm{mg} / \mathrm{l} / \mathrm{h}$ of productivity and $5436 \mathrm{mg} / \mathrm{l}$ of titer was achieved (Sun, Zhao, Xiong, Zhang, \& Bi, 2016).

\section{INCREASED PRODUCTION OF CRUDE VIOLACEIN FROM E. COLI \\ 7.1 VIA GLUCOSE ENGINEERED WITH INTERACTIVE CONTROL TRYPTOPHAN AND VIOLACEIN SYNTHETIC PATHWAY}

Fang and his co-workers conducted an experiment to engineer $E$. coli to increase the production of crude violacein by controlling trp and violacein synthetic pathways via glucose. Firstly, strains were generated that have a multivariate module for differed throughputs of trp. This was achieved by overexpression of 2 vital genes from the upstream trp metabolic pathway i.e. $\operatorname{trpE}{ }^{\text {fbr }} / \operatorname{trpD}$ genes along with conjugational knockout of 3 genes i.e. pheA/tnaA/trpR genes (fig. 12).

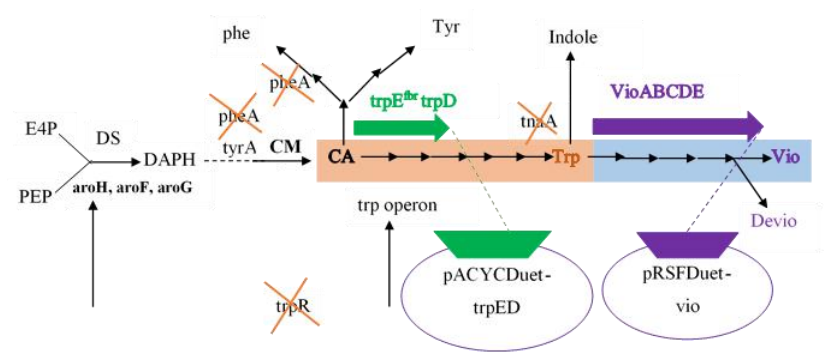

Fig.12: Plasmid construction and trp accumulation gene knockdown

To achieve this, trp metabolic pathway was thoroughly studied in C. glutamicum and E. coli. Pathway initiates with E4P and PEP condensation to form chorismate which is accompanied by trp synthesis via trp branch 
pathway. There is also the presence of trp branches and phenylalanine which force chorismate to produce tyrosine and phenylalanine. Trp repression, attenuation and feedback inhibition controlled the flow of trp metabolic pathway (Ikeda, 2006). Hence, there are 2 possible ways of generation of trp-accumulating strains i.e. either by improving metabolic pathway flux increasing key enzyme's activity in the metabolic biosynthetic pathway of chorismate and trp operon or by deletion of genes associated with trp accretion inhibition from the chromosome by repressing trp degradation (tnaA), chorismate competition to produce aromatic amino acids (pheA) and trp operon transcription (trpR) (Zhao et al., 2011) (Gu, Yang, Kang, Wang, \& Qi, 2012).

After the generation of trp biosynthetic pathway, the violacein synthetic gene cluster was instigated downstream. Maximum production of crude violacein directly via glucose was achieved in a cultural flask with a titer of $0.6 \pm 0.01 \mathrm{~g} / 1$ in $E$. coli $\mathrm{B} 2 / \mathrm{pED}+$ pVio that was $3.98 \mathrm{X}$ more than control $\mathrm{B} 1 / \mathrm{pV}$ io devoid of trp pathway upregulation i.e. $0.153 \pm 0.005 \mathrm{~g} / \mathrm{l}$ after collaborating these 2 pathways. The highest crude violacein productivity i.e. $36 \mathrm{mg} / \mathrm{h} / \mathrm{l}$ and titer $1.75 \mathrm{~g} / \mathrm{l}$ was evinced by recombinant $E$. coli $\mathrm{B} 2 / \mathrm{pED}+\mathrm{pVio}$, which was $4.48 \mathrm{X}$ more than C. freundii (pCom10vio) (Xiao et al., 2011) when subjected to the same cultural conditions devoid of the addition of trp, using C-source glucose (glucose yield $=0.116 \quad \mathrm{~g}$-violacein/g-glucose and glucose consumption $=15.1 \mathrm{~g} / \mathrm{l}$ ) in a $5 \mathrm{~L}$ bioreactor batch fermentation (Fang et al., 2015).

\subsection{VIA GLUCOSE BY OVEREXPRESSING RATE-LIMITING VITAL ENZYMES}

For the production of crude violacein using the economic industrial source of carbon, Zhou with his co-workers initiated the biosynthetic pathway of violacein in E. coli strain B8/pTRPH1, on which work had done in the previous study as described above to engineer this strain metabolically for trp accumulation via glucose. By using glucose as a carbon source in a medium, they were able to produce a higher amount of crude violacein of capacity $0.25 \mathrm{~g} / \mathrm{l} / \mathrm{OD}_{600}$. They disclosed VioE enzyme to be the rate-limiting step biosynthesis of violacein by further over-expression of all of the 5 genes i.e. vioABCDE that were associated with the biosynthetic pathways of violacein. In a $5 \mathrm{~L}$ bioreactor undergoing fed-batch fermentation, the crude violacein productivity $98.7 \mathrm{mg} / \mathrm{l} / \mathrm{h}$ along with the titer of $4.45 \mathrm{~g} / \mathrm{l}$ was produced using the optimal E. coli strain i.e. B8/pTRPH $\mathrm{H}_{1}$-pVio-VioE. It was revealed that this strain had produced the highest amount of crude violacein productivity and titer so far (Zhou, Fang, Li, Zhang, \& Xing, 2018).

\section{VIOLACEIN BIOSYNTHESIS BY ENGINEERING OLEAGINOUS YEAST YARROWIA LIPOLYTICA}

In the food industry, $Y$. lipolytica, as a host, is extensively espoused for the production of $\beta$-ionone (Czajka et al., 2018), $\beta$-carotenoids (Larroude et al., 2018) (Gao et al., 2017) and citric acid (Fickers et al., 2005). It was found that both $\mathrm{C}$. violacein and Y. lipolytica were collected from the marine surroundings that had high GC contents i.e. about $65 \%$. Scientists argue that due to the GRAS status of the $Y$. lipolytica, it can provide a novel platform for the biosynthesis of violacein. In this study, the extraction of the violacein from yeast culture was optimized in order to improve the purity and recovery ratio of violacein from the culture by keeping in consideration incubation time, using cell wall degrading enzymes as the cell wall of Y. lipolytica is composed of thick polymer i.e. chitin having galactose and mannose (Liu, Ding, Sun, Boussetta, \& Vorobiev, 2016), mechanical shear stress choice i.e. using glass beads and vortex (Jones et al., 2015) and organic solvent's variations i.e. methanol or ethyl acetate (Xu, Rizzoni, Sul, \& Stephanopoulos, 2017). The quantitative relation between the microplate reader method and HPLC proved to be equivalent to measuring the production of violacein from the yeast culture. By using the extraction protocol, maximum production of violacein and deoxyviolacein was obtained in shake flasks i.e. $70.04 \mathrm{mg} / \mathrm{l}$ and $5.28 \mathrm{mg} / \mathrm{l}$ respectively. At $60 \mathrm{C} / \mathrm{N}$ ration with the incorporation of $10 \mathrm{~g} / \mathrm{l}$ of $\mathrm{CaCO}_{3}$ in order to optimize the $\mathrm{pH}$ of the media, the purity of violacein reached $86.92 \%$ (Tong, Zhou, Zhang, \& Xu, 2019).

\section{VIOLACEIN BIOSYNTHESIS BY DUGANELLA VIOLACEINIGRA}

In this study, Choi and his collaborators isolated a violacein biosynthetic new strain that was identified to be the relative of D. violaceinigra YIM 31327 on the basis of a phylogenetic analysis by using FAME (fatty acid methyl ester) analysis, vioA and gyrB gene sequences and $16 \mathrm{~S}$ rRNA sequencing. Along with its isolation and identification, within the last few years, cloning of vioABCDE genes and its heterogeneous expression and fermentation for the biosynthesis of violacein had been reported (Rodrigues et al., 2012) (Rodrigues et al., 2013). This newly isolated strain had been identified as D. violaceinigra NI28 strain. Though the phylogenetic analysis showed similarity between these 2 strains, N128 strain showed a different phenotype than YIM 31327 strain, as it was able to grow 25\% faster than YIM 31327 on nutrient media and was able to produce $45 \mathrm{X}$ more violacein at a higher rate ( $\mathrm{Li}$ et al., 2004) (Kämpfer, Wellner, Lohse, Martin, \& Lodders, 2012). N128 was 
disclosed to be the best producer of violacein when was compared with $J$. lividum and other violacein producers. For example, at $24 \mathrm{~h}, \mathrm{~N} 128$ strain produced a yield of 6.0 $\mathrm{mg} / \mathrm{OD}$ of crude violacein, which was $2 \mathrm{X}$ more than other strains of D. violaceinigra (Choi, Kim, Lyuck, Kim, \& Mitchell, 2015).

\section{ANTI-MICROBIAL IMPACT OF VIOLACEIN ON STAPHYLOCOCCUS SPECIE}

After high-level production of violacein as discussed above, Choi et al scrutinized the antibacterial activity of crude violacein on drug-tolerant Staphylococcus aureus. It was divulged that the minimum concentration of crude violacein to undergo inhibitory effect on S. aureus was $1.8 \mu \mathrm{M}$. However, $96 \%$ loss in the initial population of $\mathrm{S}$. aureus was achieved by adding $30 \mu \mathrm{M}$ of the crude violacein (Choi et al., 2015).

Similarly, in another study conducted by Dodou et al, the anti-microbial activity of violacein was determined on $S$. epidermidis and its symbiotic impact on the antibiotics. Excellent anti-microbial effect of violacein was revealed on both non-biofilm and biofilm-forming strains of $\mathrm{S}$. epidermis i.e. ATCC 12228 and ATCC 35984 respectively. In short time period of exposure, both the bactericidal (for both strains, $\mathrm{MBC}=20 \mu \mathrm{g} / \mathrm{ml}$ ) and bacteriostatic effects (MIC $=10 \mu \mathrm{g} / \mathrm{ml}$ for ATCC 12228 and $\mathrm{MIC}=20 \mu \mathrm{g} / \mathrm{ml}$ for ATCC 35984) were observed. After exposure to $2-3 \mathrm{~h}$, the bactericidal concentration of violacein led to the death of $S$. epidermidis. In addition, the violacein symbiotically optimized the action of various antimicrobial types on $\mathrm{S}$. epidermidis strain ATCC $35984(545 \% ; n=6)$ and strain ATCC 12228 $(818 \% ; n=9)$, thus decreases the MIC up to $16 \mathrm{X}$ of these respective antibiotics (Dodou et al., 2017).

\section{ANTI-CANCEROUS ACTIVITY INDUCED BY VIOLACEIN IN THE RESISTANT LEUKEMIA CELLS}

Generally, it is recognized that the cancerous processes are intimately linked with the various modes of PCD (programmed cell death). But the problem is that there is not well-known PCD mechanism that is involved in the chemoprevention of cancer and it can differ between types of tumor cells involved and kinds of chemopreventive agents. Thus, according to pharmacologists, it is quite vital to describe the candidate's cellular specificity along with its bypass dysfunctional tumoral signaling pathway's capability to provide insensitivity to the death stimulus during the initial steps of drug development. While studying the violacein's cytotoxic effect, it was disclosed that the death that was induced in the leukemia progenitor cells i.e. $\mathrm{CD} 34^{+} / \mathrm{c}-\mathrm{Kit}^{+} / \mathrm{P}-$ glycoprotein ${ }^{+} / \mathrm{MRP}^{+}$TF1 was not mediated by autophagy or apoptosis, as this compound did not significantly affect the biomarkers of both kinds of cell death. Working mechanisms of violacein were clarified by performing kinome profiling that used peptide arrays that determined the elaborated descriptions of activities of the cellular kinase. Activation of PDK, PKA and AKT along with the inhibition of DAPK1 and calpain carried out the pro-death activity of violacein that is accompanied the structural changes that are caused by Golgi apparatus collapse and ER stress, that lead to cellular quietus. The results declared that kinome reprogramming was induced by violacein that overcame death signaling affliction of tolerant leukemia cells (Queiroz et al., 2012).

\section{CONCLUSION}

This review is basically based on the study of the production of violacein and its derivatives from metabolically engineered microorganisms. Violacein being a secondary metabolite is found to have a high range of biological activities like anti-microbial activities and anti-cancerous activities. Due to these biological activities, scientists have developed an increased emphasis to study this compound and increasing the production via both wild-type strains and recombinant microbial strains. As discussed in this review, the production of violacein and its characterization can't be achieved without its struggles and obstacles and there is still more work that can be done. This, in particular, is based on the mode of action of violacein that needs to be studied in more in detail. The current tendency in the molecular genetic field has basically helped the researchers to genetically engineered the bacterial host that can undergo the overproduction of the violacein within the fermentation. A supplementary scheme in order to enhance the production abilities of genetically engineered strain should be kept in consideration including all the aspects of gene expression, optimization of bioprocessing and downstream processing of violacein and its derivative. Generally, bioprocess optimization for heterologous product formation entails the incrementation of metabolic abilities of engineered hot for the desired compound or product. The engineered hot of violacein and deoxyviolacein possess a high flux trp pathway enciphered in their genome that acts as a strong initiating point to obtain various high-value trp-bed therapeutic. In the future, violacein and its derivative will become readily accessible for clinical studies and the scientific community. 


\section{REFERENCES}

[1] Antonio, R., Haun, M., \& Pilli, R. (1994). Biosynthesis Of A Trypanocide By Chromobacterium Violaceum. World Journal Of Microbiology \& Biotechnology.

[2] Antônio, R. V., \& Creczynski-Pasa, T. B. (2004). Genetic analysis of violacein biosynthesis by Chromobacterium violaceum. Genet Mol Res, 3(1), 85-91.

[3] Aranda, S., Montes-Borrego, M., \& Landa, B. B. (2011). Purple-pigmented violacein-producing Duganella spp. inhabit the rhizosphere of wild and cultivated olives in southern Spain. Microbial ecology, 62(2), 446-459.

[4] Asamizu, S., Kato, Y., Igarashi, Y., \& Onaka, H. (2007). VioE, a prodeoxyviolacein synthase involved in violacein biosynthesis, is responsible for intramolecular indole rearrangement. Tetrahedron letters, 48(16), 2923-2926.

[5] August, P., Grossman, T., Minor, C., Draper, M., MacNeil, I., Pemberton, J., . . . Osburne, M. (2000). Sequence analysis and functional characterization of the violacein biosynthetic pathway from Chromobacterium violaceum. Journal of molecular microbiology and biotechnology, 2(4), 513-519.

[6] Avguštin, J. A., Bertok, D. Ž., \& Avguštin, G. (2013). Isolation and characterization of a novel violacein-like pigment producing psychrotrophic bacterial species Janthinobacterium svalbardensis sp. nov. Antonie Van Leeuwenhoek, 103(4), 763-769.

[7] Balibar, C. J., \& Walsh, C. T. (2006). In vitro biosynthesis of violacein from 1-tryptophan by the enzymes VioA- E from Chromobacterium violaceum. Biochemistry, 45(51), 15444-15457.

[8] Brady, S. F., Chao, C. J., Handelsman, J., \& Clardy, J. (2001). Cloning and heterologous expression of a natural product biosynthetic gene cluster from eDNA. Organic Letters, 3(13), 1981-1984.

[9] Burt, S. A., Ojo-Fakunle, V. T., Woertman, J., \& Veldhuizen, E. J. (2014). The natural antimicrobial carvacrol inhibits quorum sensing in Chromobacterium violaceum and reduces bacterial biofilm formation at sublethal concentrations. PLoS One, 9(4), e93414.

[10] Choi, S. Y., Kim, S., Lyuck, S., Kim, S. B., \& Mitchell, R. J. (2015). High-level production of violacein by the newly isolated Duganella violaceinigra str. NI28 and its impact on Staphylococcus aureus. Scientific reports, 5, 15598.

[11] Czajka, J. J., Nathenson, J. A., Benites, V. T., Baidoo, E. E., Cheng, Q., Wang, Y., \& Tang, Y. J. (2018). Engineering the oleaginous yeast Yarrowia lipolytica to produce the aroma compound $\beta$-ionone. Microbial cell factories, 17(1), 136.

[12] Dantas, C., Volpe, P. L., Durán, N., \& Ferreira, M. (2012). The violacein biosynthesis monitored by multiwavelength fluorescence spectroscopy and by the PARAFAC method. Journal of the Brazilian Chemical Society, 23(11), 2054-2064.

[13] de Vasconcelos, A. T. R., De Almeida, D. F., Hungria, M., Guimaraes, C. T., Antônio, R. V., Almeida, F. C., . . . Andrade, E. M. (2003). The complete genome sequence of Chromobacterium violaceum reveals remarkable and exploitable bacterial adaptability. Proceedings of the national academy of sciences of the United States of America, 11660-11665.

[14] Dodou, H., de Morais Batista, A., Sales, G., de Medeiros, S., Rodrigues, M., Nogueira, P., . . Nogueira, N. (2017). Violacein antimicrobial activity on Staphylococcus epidermidis and synergistic effect on commercially available antibiotics. Journal of applied microbiology, 123(4), 853-860.

[15] Durán, N., Justo, G. Z., Ferreira, C. V., Melo, P. S., Cordi, L., \& Martins, D. (2007). Violacein: properties and biological activities. Biotechnology and applied biochemistry, 48(3), 127-133.

[16] Durán, N., \& Menck, C. F. (2001). Chromobacterium violaceum: a review of pharmacological and industiral perspectives. Critical reviews in microbiology, 27(3), 201222.

[17] Ellis, G. A., Tschirhart, T., Spangler, J., Walper, S. A., Medintz, I. L., \& Vora, G. J. (2019). Exploiting the Feedstock Flexibility of the Emergent Synthetic Biology Chassis Vibrio natriegens for Engineered Natural Product Production. Mar Drugs, 17(12). doi: 10.3390/md17120679

[18] Fang, M.-Y., Zhang, C., Yang, S., Cui, J.-Y., Jiang, P.-X., Lou, K., . . . Xing, X.-H. (2015). High crude violacein production from glucose by Escherichia coli engineered with interactive control of tryptophan pathway and violacein biosynthetic pathway. Microbial cell factories, 14(1), 8.

[19] Fickers, P., Benetti, P.-H., Waché, Y., Marty, A., Mauersberger, S., Smit, M., \& Nicaud, J.-M. (2005). Hydrophobic substrate utilisation by the yeast Yarrowia lipolytica, and its potential applications. FEMS yeast research, 5(6-7), 527-543.

[20] Gao, S., Tong, Y., Zhu, L., Ge, M., Zhang, Y., Chen, D., . . . Yang, S. (2017). Iterative integration of multiple-copy pathway genes in Yarrowia lipolytica for heterologous $\beta$ carotene production. Metabolic engineering, 41, 192-201.

[21] Gu, P., Yang, F., Kang, J., Wang, Q., \& Qi, Q. (2012). One-step of tryptophan attenuator inactivation and promoter swapping to improve the production of Ltryptophan in Escherichia coli. Microbial cell factories, 11(1), 30.

[22] Heery, D. M., Fitzpatrick, R., \& Dunican, L. K. (1994). A Sequence from a Tryptophan-Hyperproducing Strain of Corynebacterium glutamicum Encoding Resistance to 5Methyltryptophan. Biochemical and Biophysical Research Communications, 201(3), 1255-1262. doi: https://doi.org/10.1006/bbrc.1994.1840

[23] Hillson, N. J., Rosengarten, R. D., \& Keasling, J. D. (2012). j5 DNA assembly design automation software. ACS synthetic biology, 1(1), 14-21.

[24] Hirano, S., Asamizu, S., Onaka, H., Shiro, Y., \& Nagano, S. (2008). Crystal structure of VioE, a key player in the construction of the molecular skeleton of violacein. Journal of Biological Chemistry, 283(10), 6459-6466.

[25] Hoshino, T., \& Ogasawara, N. (1990). Biosynthesis of violacein: evidence for the intermediacy of 5-hydroxy-Ltryptophan and the structure of a new pigment, 
oxyviolacein, produced by the metabolism of 5hydroxytryptophan. Agricultural and biological chemistry, 54(9), 2339-2346.

[26] Huang, Y., Lai, X., He, X., Cao, L., Zeng, Z., Zhang, J., \& Zhou, S. (2009). Characterization of a deep-sea sediment metagenomic clone that produces water-soluble melanin in Escherichia coli. Marine biotechnology, 11(1), 124-131.

[27] Ikeda, M. (2006). Towards bacterial strains overproducing L-tryptophan and other aromatics by metabolic engineering. Applied microbiology and biotechnology, 69(6), 615.

[28] Ikeda, M., \& Katsumata, R. (1999). Hyperproduction of Tryptophan byCorynebacterium glutamicum with the Modified Pentose Phosphate Pathway. Applied and Environmental Microbiology, 65(6), 2497-2502.

[29] Jakoby, M., Krämer, R., \& Burkovski, A. (1999). Nitrogen regulation in Corynebacterium glutamicum: isolation of genes involved and biochemical characterization of corresponding proteins. FEMS Microbiology Letters, 173(2), 303-310. doi: https://doi.org/10.1016/S0378-1097(99)00085-3

[30] Jiang, P.-x., Wang, H.-s., Xiao, S., Fang, M.-y., Zhang, R.-p., He, S.-y., . . Xing, X.-H. (2012). Pathway redesign for deoxyviolacein biosynthesis in Citrobacter freundii and characterization of this pigment. Applied Microbiology and Biotechnology, 94(6), 1521-1532.

[31] Jiang, P.-x., Wang, H.-s., Zhang, C., Lou, K., \& Xing, X.H. (2010). Reconstruction of the violacein biosynthetic pathway from Duganella sp. B2 in different heterologous hosts. Applied Microbiology and Biotechnology, 86(4), 1077-1088.

[32] Jones, J. A., Vernacchio, V. R., Lachance, D. M., Lebovich, M., Fu, L., Shirke, A. N., . . Koffas, M. A. (2015). ePathOptimize: a combinatorial approach for transcriptional balancing of metabolic pathways. Scientific reports, 5, 11301.

[33] Kämpfer, P., Wellner, S., Lohse, K., Martin, K., \& Lodders, N. (2012). Duganella phyllosphaerae sp. nov., isolated from the leaf surface of Trifolium repens and proposal to reclassify Duganella violaceinigra into a novel genus as Pseudoduganella violceinigra gen. nov., comb. nov. Systematic and applied microbiology, 35(1), 19-23.

[34] Larroude, M., Celinska, E., Back, A., Thomas, S., Nicaud, J. M., \& Ledesma-Amaro, R. (2018). A synthetic biology approach to transform Yarrowia lipolytica into a competitive biotechnological producer of $\beta$-carotene. Biotechnology and bioengineering, 115(2), 464-472.

[35] Lee, H. H., Ostrov, N., Wong, B. G., Gold, M. A., Khalil, A. S., \& Church, G. M. (2019). Functional genomics of the rapidly replicating bacterium Vibrio natriegens by CRISPRi. Nature microbiology, 4(7), 1105-1113.

[36] Li, W.-J., Zhang, Y.-Q., Park, D.-J., Li, C.-T., Xu, L.-H., Kim, C.-J., \& Jiang, C.-L. (2004). Duganella violaceinigra sp. nov., a novel mesophilic bacterium isolated from forest soil. International journal of systematic and evolutionary microbiology, 54(5), 1811-1814.

[37] Liu, D., Ding, L., Sun, J., Boussetta, N., \& Vorobiev, E. (2016). Yeast cell disruption strategies for recovery of intracellular bio-active compounds-A review. Innovative food science \& emerging technologies, 36, 181-192.

[38] McClean, K. H., Winson, M. K., Fish, L., Taylor, A., Chhabra, S. R., Camara, M., . . Bycroft, B. W. (1997). Quorum sensing and Chromobacterium violaceum: exploitation of violacein production and inhibition for the detection of N-acylhomoserine lactones. Microbiology, 143(12), 3703-3711.

[39] Momen, A. R., \& Hoshino, T. (2000). Biosynthesis of violacein: intact incorporation of the tryptophan molecule on the oxindole side, with intramolecular rearrangement of the indole ring on the 5-hydroxyindole side. Bioscience, biotechnology, and biochemistry, 64(3), 539-549.

[40] Pühler, A., Kalinowski, J., \& Tauch, A. (2008). Amino acid production by Corynebacterium glutamicum in the light of modern genome research. Journal of Biotechnology, 136, S279. doi: https://doi.org/10.1016/j.jbiotec.2008.07.597

[41] Queiroz, K. C., Milani, R., Ruela-de-Sousa, R. R., Fuhler, G. M., Justo, G. Z., Zambuzzi, W. F., . . Ferreira, C. V. (2012). Violacein induces death of resistant leukaemia cells via kinome reprogramming, endoplasmic reticulum stress and Golgi apparatus collapse. PLoS One, 7(10), e45362.

[42] Rodrigues, A. L., Göcke, Y., Bolten, C., Brock, N. L., Dickschat, J. S., \& Wittmann, C. (2012). Microbial production of the drugs violacein and deoxyviolacein: analytical development and strain comparison. Biotechnology letters, 34(4), 717-720.

[43] Rodrigues, A. L., Trachtmann, N., Becker, J., Lohanatha, A. F., Blotenberg, J., Bolten, C. J., . . Sprenger, G. A. (2013). Systems metabolic engineering of Escherichia coli for production of the antitumor drugs violacein and deoxyviolacein. Metabolic engineering, 20, 29-41.

[44] Ryan, K. S., Balibar, C. J., Turo, K. E., Walsh, C. T., \& Drennan, C. L. (2008). The violacein biosynthetic enzyme VioE shares a fold with lipoprotein transporter proteins. Journal of Biological Chemistry, 283(10), 6467-6475.

[45] Sánchez, C., Braña, A. F., Méndez, C., \& Salas, J. A. (2006). Reevaluation of the violacein biosynthetic pathway and its relationship to indolocarbazole biosynthesis. Chembiochem, 7(8), 1231-1240.

[46] Schloss, P. D., Allen, H. K., Klimowicz, A. K., Mlot, C., Gross, J. A., Savengsuksa, S., . . Handelsman, J. (2010). Psychrotrophic strain of Janthinobacterium lividum from a cold Alaskan soil produces prodigiosin. DNA and cell biology, 29(9), 533-541.

[47] Sun, H., Zhao, D., Xiong, B., Zhang, C., \& Bi, C. (2016). Engineering Corynebacterium glutamicum for violacein hyper production. Microbial cell factories, 15(1), 148.

[48] Tauch, A., Kirchner, O., Löffler, B., Götker, S., Pühler, A., \& Kalinowski, J. (2002). Efficient electrotransformation of Corynebacterium diphtheriae with a mini-replicon derived from the Corynebacterium glutamicum plasmid pGA1. Current microbiology, 45(5), 362-367.

[49] Tong, Y., Zhou, J., Zhang, L., \& Xu, P. (2019). Engineering oleaginous yeast Yarrowia lipolytica for 
violacein production: extraction, quantitative measurement and culture optimization. bioRxiv, 687012.

[50] Wang, H., Wang, F., Zhu, X., Yan, Y., Yu, X., Jiang, P., \& Xing, X.-H. (2012). Biosynthesis and characterization of violacein, deoxyviolacein and oxyviolacein in heterologous host, and their antimicrobial activities. Biochemical engineering journal, 67, 148-155.

[51] Wang, Y., Ikawa, A., Okaue, S., Taniguchi, S., Osaka, I., Yoshimoto, A., .. Enomoto, K. (2008). Quorum sensing signaling molecules involved in the production of violacein by Pseudoalteromonas. Bioscience, biotechnology, and biochemistry, 72(7), 1958-1961.

[52] Waters, C. M., \& Bassler, B. L. (2005). Quorum sensing: cell-to-cell communication in bacteria. Annu. Rev. Cell Dev. Biol., 21, 319-346.

[53] Williams, P., Winzer, K., Chan, W. C., \& Camara, M. (2007). Look who's talking: communication and quorum sensing in the bacterial world. Philosophical Transactions of the Royal Society B: Biological Sciences, 362(1483), 1119-1134.

[54] Xiao, S., Yang, C., Jiang, P., Zhang, C., Lou, K., \& Xing, X.-H. (2011). Fed-batch fermentation of recombinant Citrobacter freundii with expression of a violaceinsynthesizing gene cluster for efficient violacein production from glycerol. Biochemical engineering journal, 57, 5562.

[55] Xu, P., Rizzoni, E. A., Sul, S.-Y., \& Stephanopoulos, G. (2017). Improving metabolic pathway efficiency by statistical model-based multivariate regulatory metabolic engineering. ACS synthetic biology, 6(1), 148-158.

[56] Yada, S., Wang, Y., Zou, Y., Nagasaki, K., Hosokawa, K., Osaka, I., . . . Enomoto, K. (2008). Isolation and characterization of two groups of novel marine bacteria producing violacein. Marine biotechnology, 10(2), 128132.

[57] Yang, Y.-T., Bennett, G. N., \& San, K.-Y. (1998). Genetic and metabolic engineering. Electronic Journal of Biotechnology, 1(3), 20-21.

[58] ZHANG, X. (2010). Violacein biosynthesis and its regulation in Pseudoalteromonas sp. 520P1.

[59] Zhao, M., Ma, Y., Wei, Z.-z., Yuan, W.-X., Li, Y.-1., Zhang, C.-h., . . . Zhou, H.-j. (2011). Determination and comparison of $\gamma$-aminobutyric acid (GABA) content in pu-erh and other types of Chinese tea. Journal of agricultural and food chemistry, 59(8), 3641-3648.

[60] Zhou, Y., Fang, M.-Y., Li, G., Zhang, C., \& Xing, X.-H. (2018). Enhanced production of crude violacein from glucose in Escherichia coli by overexpression of ratelimiting key enzyme (s) involved in violacein biosynthesis. Applied biochemistry and biotechnology, 186(4), 909-916. 